C. APARICIO**

F. J. GIL**

A. PADRÓ ${ }^{*}$

C. PERAIRE***

J. A. PLANELL ${ }^{* * *}$

* Clínica Padrós. Barcelona.

** Departamento de Ciencias de Materiales e Ingeniería Metalúrgica. E.T.S. Ingenieros Industriales de Barcelona.

Universidad Politécnica de Cataluña. Diagonal 647. 08028 Barcelona.

*** CID. A.S.A.L.

Centro de Investigación y Desarrollo Aplicado S.A.L.

Santa Perpètua de la Mogoda.

Barcelona.

\section{Influencia de la naturaleza de las partículas de proyección en el proceso de shot peening en la mejora de la osteointegración de implantes dentales}

\section{Influence of the nature of the projection particles used in the shot peening process on dental-implant osteointegration}

Resumen

El presente trabajo se ha centrado en valorar el efecto que tiene sobre las características superficiales, tratar la superficie de discos de Ti C.P., mediante un shot peening con distintos tipos de partículas de proyección: $\mathrm{Al}_{2} \mathrm{O}_{3}, \mathrm{TiO}_{2}$ y SiC. También se ha determinado la influencia que tiene pasivar los discos tratados con $\mathrm{SiC}$, con $\mathrm{HCl}$ o con $\mathrm{HF} / \mathrm{HNO}_{3}$; sobre todo desde el punto de vista de la limpieza superficial después del tratamiento, ya que se pretende aprovechar la reacción química que se produce entre el SiC y el HF, con la formación de SiF. Para todo ello se han efectuado ensayjos de la rugosidad superficial de los discos después del shot peening; tanto cuantitativos como cualitativos. Asimismo se ha determinado la capacidad de la partícula de soportar el shot peening sin perder su característica más importante dentro del proceso: su tamaño inicial. Finalmente, se ha propuesto un proceso de fabricación de partículas de $\mathrm{TiO}_{2}$ por sinterización. La fabricación en el laboratorio ha sido necesaria debido a la imposibilidad de encontrar en el mercado las partículas de esta naturaleza.

Palabras clave: Osteointegración. Implantes. Shot peening

\section{Summary}

An evaluation was made of the effect on surface properties on superficial treatment of Ti C.P. by shot peening using different types of projection particles: $\mathrm{Al}_{2}, \mathrm{O}_{3}, \mathrm{TiO}_{2}$, and $\mathrm{SiC}$. The influence of deactivating $\mathrm{SiC}_{3}$ treated disks with $\mathrm{HCl}$ or $\mathrm{HF} / \mathrm{HNO}_{3}$ also was examined in relation to surface cleaning after treatment, based on taking advantage of the chemical reaction between $\mathrm{SiC}$ and $\mathrm{HF}$ to form $\mathrm{SiF}_{4}$. Quantitative and qualitative tests were made of the surface roughness of disks after shot peening. Likewise, particle capacity to withstand shot peening without losing their most important property, initial size, also was evaluated. Finally, a process of fabricating $\mathrm{TiO}_{2}$ particles by sinterization was proposed. Laboratory fabrication was necessary because particles of this type are not available commercially.

Key words: Osteointegration. Implants, Shot peening.

Correspondencia

F. J. GIL. Departamento de Ciencias de Materiales e Ingeniería Metalúrgica. E.T.S. Ingenieros Industriales de Barcelona. Universidad Politécnica de Cataluna. Diagonal 647. Tel.: (93) 40167 08. Fax: (93) 4016706. 08028 Barcelona. 


\section{Introducción}

Una de las variables que se sabe que influye de forma determinante en el buen comportamiento del implante dental de titanio comercialmente puro (Ti C.P.), tanto a corto como a largo plazo, es su rugosidad superficial. En este sentido, se han realizado estudios in vivo que demuestran que una cierta rugosidad superficial mejora la fijación del implante al hueso ${ }^{1-3}$. Por otra parte, estudios in vitro han puesto de manifiesto que la diferenciación y proliferación osteoblástica, así como la producción de matriz ósea, también resultan influenciadas positivamente por el aumento de la rugosidad de la superficie ${ }^{4-6}$

Un procedimiento ya estudiado y utilizado para incrementar la rugosidad en la superficie de los implantes dentales es el shot peening o blasting. Este procedimiento que consiste en proyectar partículas de elevada dureza a gran velocidad y presión sobre la superficie del implante es adecuado porque además de dar rugosidad superficial, limpia la superficie de contaminantes; debido al aumento de la tensión compresiva en las primeras capas del substrato, aumenta la vida a fatiga del implante; y ennoblece la superficie y, por lo tanto, aumenta su resistencia a la corrosión ${ }^{7}$

Una de las variables más importantes del shot peening es la naturaleza de las partículas utilizadas para ser proyectadas contra la superficie del metal a tratar. En primer lugar, esta importancia viene determinada por el hecho de que después del tratamiento superficial, limpieza, pasivado y esterilización del implante, siempre quedan restos de partículas procedentes del shot peening que, con posterioridad a la implantación, pueden desprenderse y pasar a los tejidos circundantes. En este sentido, Gross y Strunz ${ }^{8}$ concluyeron que la cesión de partículas de $\mathrm{Al}_{2} \mathrm{O}_{3}$, uno de los tipos más utilizados como partículas de proyección, podía entorpecer la mineralización normal del hueso. Para evitarlo se puede, por ejemplo, proyectar partículas de la misma composición química que la superficie del implante, es decir, $\mathrm{TiO}_{2}$. También se pueden buscar otras naturalezas que influyan menos negativamente o que presenten la posibilidad de eliminarse de la superficie del implante con más facilidad.

Por otro lado, la naturaleza de la partícula es también relevante por la capacidad que cada una tiene para realizar el trabajo mecánico, sobre la superficie del implante, necesario para conseguir la rugosidad deseada.

Por estos dos motivos, se hace necesario investigar la influencia que el tipo de partícula de proyección va a tener sobre las características de los implantes den-
tales de Titanio C.P.

\section{Materiales y métodos}

\section{Preparación de los discos}

Para la realización de los diferentes estudios se prepararon discos de $6 \mathrm{~mm}$ de diámetro y un espesor de
$4 \mathrm{~mm}$, fabricados de Ti C.P. Se les ha realizado un tratamiento superficial de shot peening, con una presión de chorro de $0,25 \mathrm{MPa}$, durante $5 \mathrm{~s}$.

Se han preparado distintos grupos de discos en función del tipo de partícula de proyección: $\mathrm{Al}_{2} \mathrm{O}_{3}$, $\mathrm{TiO}_{2}$ y SiC; y en el caso del SiC también se han diferenciado discos con tratamiento de pasivado realizado con solución de $\mathrm{HCl}$ o con solución de $\mathrm{HF} / \mathrm{HNO}_{3}$. Los discos chorreados con óxido de aluminio y los tratados con óxido de titanio se han pasivado con $\mathrm{HCl}$. En todos los casos se han utilizado partículas de gran tamaño. Después del pasivado, los discos se han esterilizado mediante óxido de etileno.

\section{Sinterización de óxido de titanio}

En este trabajo se ha fabricado partículas de $\mathrm{TiO}_{2}$ con el fin de que los residuos que se puedan quedar en la superficie del implante sean lo más inertes y lo más parecidos al material substrato.

Un total de $1.800 \mathrm{~g}$ de polvo de $\mathrm{TiO}_{2}$ (fase rutilo) químicamente puro, se han sinterizado en un horno mufla, con la siguiente pauta:

1. Se llenan 9 crisoles de alúmina con $200 \mathrm{~g}$ de polvo de $\mathrm{TiO}_{2}$ cada uno.

2. Se comprime el polvo dentro del crisol con la ayuda de una maza.

3. Dos a dos, los crisoles se introducen en el horno a $600^{\circ} \mathrm{C}$.

4. Se sube la temperatura hasta $1.000^{\circ} \mathrm{C}$, y se mantiene durante $13 \mathrm{~h}$.

5. Transcurrido dicho tiempo, se extraen los crisoles y se dejan enfriar al aire desde los $1.000^{\circ} \mathrm{C}$.

De cada crisol se ha obtenido un bloque sinterizado de óxido de titanio. Para obtener las partículas definitivas, cada bloque se ha disgregado hasta tamaños reducidos de aproximadamente $30 \mathrm{~g}$; y estos se han triturado con maza y mortero.

El producto triturado se ha cribado con tamices normalizados hasta conseguir partículas del tamaño deseado.

\section{Tamaño de partícula}

El ensayo de determinación de tamaño de partícula se ha realizado para una muestra de cada uno de los tipos de partícula $\left(\mathrm{Al}_{2} \mathrm{O}_{3}, \mathrm{TiO}_{2}\right.$ y $\mathrm{SiC}$ ); antes (SIN USAR) y después (USADO) del tratamiento de shot peening.

La determinación del tamaño de partícula se ha llevado a cabo por el método de difracción de rayos láser con un analizador «Microtrac SRA-150॰ (Leeds \& Northrup, Reino Unido)».

Las condiciones más importantes del ensayo han 
- Intensidad del rayo láser: 1,007 eV;

- Tiempo de ensayo: 30 s, y

- Número de ensayos por muestra: 3

El resultado obtenido del analizador para cada muestra es la media de los tres ensayos.

\section{Rugosidad}

Los ensayos de rugosidad superficial sobre los discos tratados se han realizado con un rugosímetro para medidas bidimensionales, con palpador de diamante de forma cónica $\left(90^{\circ}\right)$ con $5 \mu \mathrm{m}$ de diámetro en la punta, de una resolución mínima de 0,1 $\mu \mathrm{m}$, «Surftest SV-500 ${ }^{\odot}$ (Mitutoyo, Japón)». Los valores numéricos de los diferentes parámetros de rugosidad superficial, se calcularon con el software «Surfpack v3.00

(Mitutoyo, Japón)».

Se han analizado 5 discos de cada uno de los tipos implantados, realizándose 3 lecturas de rugosidad para cada uno. Para cada una de las lecturas, se ha tomado una longitud de muestra de $0,8 \mathrm{~mm}$, analizándose 3 muestras, es decir, la longitud total de ensayo para cada lectura ha sido de $2,4 \mathrm{~mm}(0,8 \mathrm{~mm} \times 3)$ Además se ha ensayado con un «pre-travel» de 0,4 mm para estabilizar la posición del disco antes de iniciar la lectura.

Se ha utilizado un filtro Gaussiano con longitud de corte igual a la longitud de muestra, 0,8 mm. Así, partiendo del perfil que detecta el rugosímetro, se filtran las longitudes de onda superiores a la de corte, obteniendo como resultado el perfil de rugosidad $R$, a partir del cual se calculan los parámetros.

Los parámetros obtenidos en cada lectura han sido:

- Ra (Media aritmética de las desviaciones del perfil): es la media aritmética del valor absoluto de las distancias desde la línea media al perfil $\mathrm{R}$ dentro de la longitud de muestra. Esta línea media es una línea de referencia para el cálculo de los distintos parámetros, que se determina por el método de los mínimos cuadrados respecto al perfil $\mathrm{R}$ en cada longitud de muestra. Ra se calcula en micrómetros y es el parámetro más general y comúnmente utilizado para describir la rugosidad.

- Rq (Media de la raíz cuadrada de las desviaciones del perfil): es el valor medio de la raíz cuadrada de las desviaciones del perfil respecto a la línea media, dentro de la longitud de muestra. Este parámetro es más sensible que Ra a los valores extremos debido a la operación de la raíz cuadrada. Rq tiene un significado estadístico ya que es la desviación estándar de la distribución de alturas del perfil.

- Ry (Máxima altura del perfil): es un valor extremo, y describe la distancia entre la altura del máximo pico y la profundidad del mayor valle, dentro de la longitud de muestra.
- Rz (Altura de diez puntos irregulares): es el valor medio, en micrómetros, del valor absoluto de los cinco picos más altos, más el valor medio del valor absoluto de los cinco valles más profundos, dentro de la longitud de muestra. Este parámetro es sensible a los cambios pronunciados en las características topográficas.

- Pc (Número de picos): es el número de pares pico-valle (ciclos) por unidad de longitud $(1 \mathrm{~cm})$ a lo largo de la línea media del perfil dentro de la longitud de muestra. Dos líneas (niveles de cuenteo) que son paralelas a la línea media se dibujan por encima y por debajo de ella a una distancia que es, en nuestro caso, el 5\% del valor del máximo pico o valle. Cada ciclo del perfil entre intersecciones del perfil y la línea media, en los que un pico cae por encima de la línea de cuenteo superior y un valle adyacente cae por debajo de la línea de cuenteo inferior, se cuenta como un ciclo pico-valle.

- HSC (Número de puntos altos): es el número de picos por unidad de longitud $(1 \mathrm{~cm})$ a lo largo de la línea media del perfil dentro de la longitud de muestra. Una línea (nivel de cuenteo), que es paralela a la línea media, se dibuja por encima de ella a una distancia que es, en nuestro caso, el 5\% del valor del máximo pico. Cada pico local que se sitúa por encima del nivel de cuenteo, es un punto alto.

- Sm (Distancia media de las irregularidades del perfil): es igual a la longitud de onda media de los ciclos pico-valle. Es el recíproco de Pc.

Las imágenes de la superficie de los discos, para realizar análisis cualitativos sobre el aspecto rugoso de las muestras, se han obtenido con la ayuda de un microscopio electrónico de barrido (M.E.B.) «JSM $6400^{\circ}$ (Jeol, Japón)». Las fotografías obtenidas muestran la superficie de los discos amplificada x200.

\section{Composición superficial}

Para analizar la composición superficial, y por lo tanto la posible contaminación de la superficie de los discos por las partículas del shot peening, se ha utilizado un analizador de dispersión de energías de rayos X, «Analytical LZ-5 (Link, Reino Unido)». Se ha analizado la cantidad porcentual atómica de los elementos más significativos para cada muestra, con ensayos de 100 segundos de duración y $20 \mathrm{KV}$ de energía del haz de rayos $X$.

\section{Crecimiento osteoblástico}

Los discos se sembraron con una suspensión celular de 12.100 células $/ \mathrm{cm}^{2}$, y se incubaron a $37^{\circ} \mathrm{C}$ con medio completo en atmósfera húmeda conteniendo 
un $5,5 \%$ de $\mathrm{CO}_{2}$. Como control negativo se utilizaron discos de poliestireno. Al cabo de 24 horas a la mitad de las muestras (grupo A) se cambió el medio de culdelipidado $200 \mu \mathrm{l}$ del medio: IMDM con un 3\% de suero tamina $C$ con carbón activado con vitamina $K_{1}$ y vitamina C. Las muestras restantes (grupo B) recibieron ducción de osteocalcina.

Tras 72 horas de incubación se recolectó el medio de cultivo de cada uno de las muestras en tubos epcontivos y se realizó un contaje celular, para ello se leyeron 5 campos de cada muestra y se obtuvo el valor medio. Se determinaron los niveles de osteocalcina. Para la calibración las concentraciones utilizadas fueron de: $0,25,0,50,1,2,4,8$ y $16 \mathrm{ng}$ de osteocalcina/ml) valorando las muestras por duplicado Este protocolo experimental se ha basado en la norma NF $590-702^{9}$ y en los estudios descritos por Clover y Gowen de $1994^{10}$

\section{Análisis estadísticos}

Todos los datos estadísticos, incluidos los valores-p en los test de comparación de medias por la t-Student y de análisis de la varianza (tabla ANOVA), se han

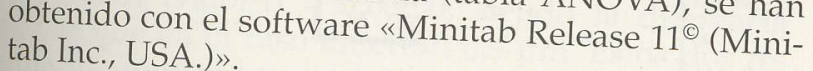

\section{Resultados y discusión}

\section{Sinterización de óxido de titanio}

El proceso de fabricación de partículas por sinterización de polvo de $\mathrm{TiO}_{2}$, ofreció el siguiente resultado: de cada $1.800 \mathrm{~g}$ de polvo introducidos en el horno, se obtuvieron aproximadamente $200 \mathrm{~g}$ de partículas con el tamaño deseado. Esto significa un rendimiento
muy pobre, de sólo un $11 \%$.

A este bajo rendimiento, se añade lo laborioso del proceso (por lento, incómodo e incluso perjudicial para la salud) y, sobre todo, las pobres características mecánicas que han demostrado estas partículas como ponen de manifiesto los ensayos de rotura de partícula y de rugosidad superficial que más adelante se

\section{Rotura de partícula}

En la tabla I se muestran los porcentajes de rotura de partícula, calculados a partir de las medidas de diámetro medio antes y después de ser usadas en el proceso de shot peening.

A la vista de los resultados se aprecia que las partículas que más se rompen son las del óxido de titanio más del doble que las de carburo de silicio y casi siete veces más que las de alúmina. Esto refuerza los co-
Tabla I. \% de rotura de los tres tipos de partículas utilizados para realzar el shot peening

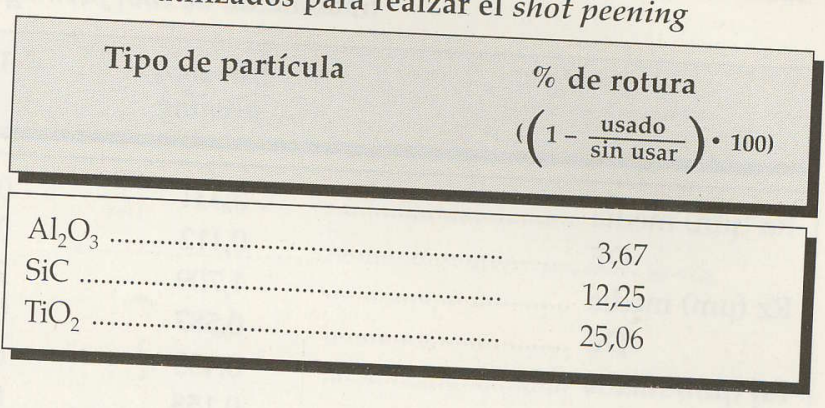

mentarios anteriores y aporta un dato significativo: las partículas de carburo de silicio son más frágiles que las de óxido de aluminio; hecho importante so de shot peening.

\section{Rugosidad}

Ra es el parámetro que se emplea habitualmente para valorar el efecto de la rugosidad de la superficie de los implantes en la respuesta biológica y funcional de los mismos; por este motivo los análisis de comparación entre los distintos tratamientos se describen y se caracterizan con él. El resto de parámetros no han aportado diferencias en las conclusiones obtenidas con $\mathrm{Ra}$, por lo tanto, su análisis no queda recogido en el presente estudio.

Los valores de la tabla II demuestran lo que es evidente a la vista de los perfiles de rugosidad (figs. 1, 2 3,4 y 5) y del diagrama de distribución de valores de Ra de la figura 6: la rugosidad superficial obtenida en $\mu \mathrm{m})$ es claramente el shot peening con $\mathrm{TiO}_{2}(\mathrm{Ra}=0,54$ con $\mathrm{Al}_{2} \mathrm{O}_{3}(\mathrm{Ra}=4,42$ inferior a la obtenida trabajando con $\mathrm{Al}_{2} \mathrm{O}_{3}(\mathrm{Ra}=4,42 \mu \mathrm{m})$, con SiC-HCl $(\mathrm{Ra}=5,00 \mu \mathrm{m})$ o con $\mathrm{SiC}-\mathrm{HF} / \mathrm{HNO}_{3}(\mathrm{Ra}=5,3 \mu \mathrm{m})$. Este resultado es contradictorio con otros trabajos en los que se ha utilizado el $\mathrm{TiO}_{2}$ como material de proyección en el shot peening ${ }^{11-13}$, y en los que no ha habido grandes diferencias en los resultados de rugosidad conseguidos entre implantes tratados con $\mathrm{Al}_{2} \mathrm{O}_{3}$ e implantes tratados con de $\mathrm{O}_{2}$. Es de reseñar, que en estos trabajos, los tamaños que los del presente estun sido mucho más pequeños método de fabriente estudio. Es evidente, pues, que el de gran da finterización, de partículas presente estudio de $\mathrm{TiO}_{2}$, que se ha llevado a cabo en el es adecuado para su utilización obstante írial de proyección en el shot peening. No ferencia en la rugosidad efecto mecánico, ya que la dito ( $\mathrm{Ra}=0,33 \mathrm{\mu m}$ ) y mente significativa (valorver en la tabla III. Además $=0,0000$ ), como se puede tenido, es super. Ademas, incluso el valor medio ob0,508 ) fijación y crecimieron Predecki y cols. ${ }^{1}$, para que haya fijación y crecimiento del hueso hacia el implante. 
Tabla II. Caracterización de la rugosidad superficial de discos de Ti (C.P.) en función de la partícula de proyección en el tratamiento de shot peening. (D.E. = Desviación Estándar)

\begin{tabular}{|c|c|c|c|c|c|}
\hline & $\begin{array}{l}\text { Sin shot } \\
\text { peening }\end{array}$ & $\mathrm{TiO}_{2}$ & $\mathrm{Al}_{2} \mathrm{O}_{3}$ & $\mathrm{SiC}-\mathrm{HCl}$ & $\mathrm{SiC}-\mathrm{HF} / \mathrm{HNO}_{3}$ \\
\hline $\operatorname{Ra}(\mu \mathrm{m})$ media & 0,331 & 0,540 & 4,423 & 4,995 & 5,293 \\
\hline 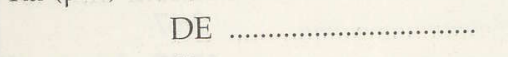 & 0,112 & 0,109 & 0,356 & 0,631 & 0,653 \\
\hline Rz $(\mu \mathrm{m})$ media & 1,709 & 2,961 & 17,191 & 18,532 & 18,502 \\
\hline DE & 0,587 & 0,617 & 0,678 & 2,116 & 1,540 \\
\hline $\mathrm{Rq}(\mu \mathrm{m})$ media & 0,455 & 0,727 & 5,489 & 6,249 & 6,540 \\
\hline DE & 0,158 & 0,169 & 0,497 & 0,787 & 0,807 \\
\hline Ry $(\mu \mathrm{m})$ media & 3,063 & 4,713 & 26,167 & 29,406 & 30,340 \\
\hline DE & 1,047 & 1,539 & 3,278 & 3,559 & 4,750 \\
\hline Pc $(/ \mathrm{cm})$ media & 150,9 & 202,6 & 82,4 & 77,2 & 67,1 \\
\hline DE & 69,1 & 121,9 & 10,3 & 9,5 & 11,2 \\
\hline HSC $(/ \mathrm{cm})$ media & 214,3 & 276,4 & 97,9 & 87,0 & 82,1 \\
\hline DE & 85,8 & 133,4 & 13,4 & 17,5 & 11,8 \\
\hline $\operatorname{Sm}(\mu \mathrm{m})$ media & 86,2 & 63,8 & 131,0 & 135,3 & 162,4 \\
\hline DE & 25,5 & 24,9 & 16,2 & 16,9 & 29,1 \\
\hline
\end{tabular}

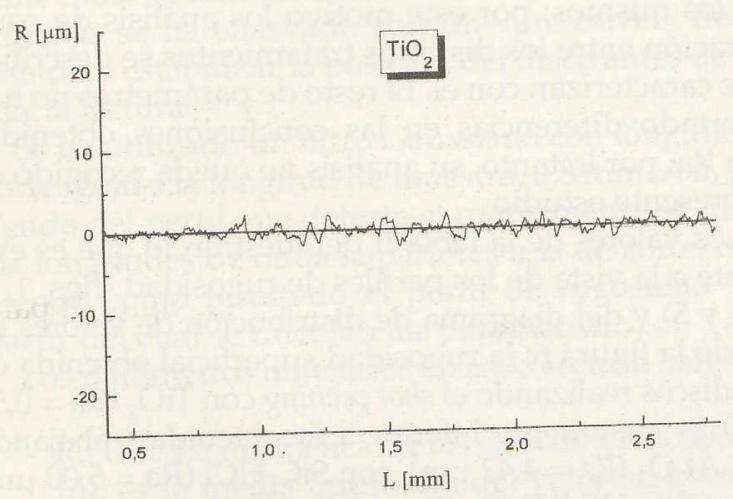

FIG. 1.-Perfil de rugosidad característico de un disco de Ti C.P. tratado con shot peening con partículas de $\mathrm{TiO}_{2}$.

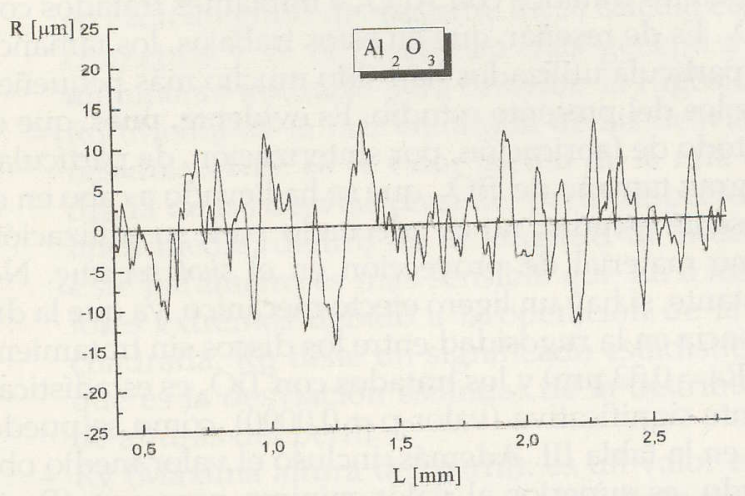

FIG. 2.-Perfil de rugosidad característico de un disco de Ti C.P. tratado con shot peening con partículas de $\mathrm{Al}_{2} \mathrm{O}_{3}$.

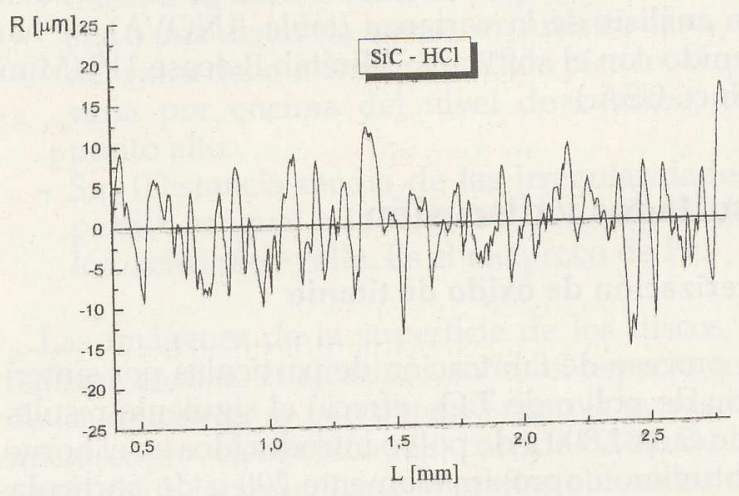

FIG. 3.-Perfil de rugosidad característico de un disco de Ti C.P. tratado con shot peening con partículas de SiC y tratamiento de pasivado con $\mathrm{HCl}$.

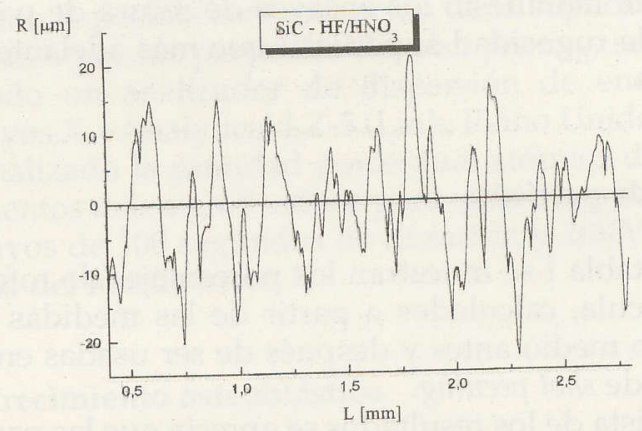

FIG. 4.-Perfil de rugosidad característico de un disco de Ti C.P. tratado con shot peening con partículas de $\mathrm{SiC}$ y tratamiento pasivado con $\mathrm{HF} / \mathrm{HNO}_{3}$. 


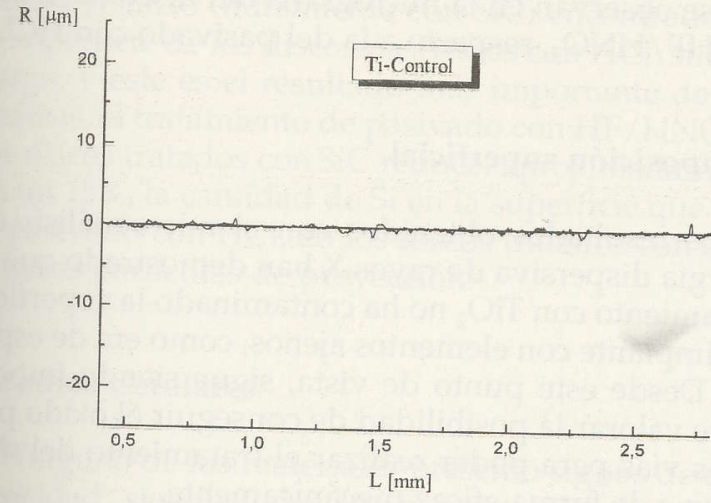

FIG. 5.-Perfil de rugosidad característico de un disco de Ti C.P. sin tratamiento con shot peening..

Por otra parte, se observa que la diferencia entre la rugosidad superficial conseguida con $\mathrm{Al}_{2} \mathrm{O}_{3}$ y $\mathrm{SiC}$ $\mathrm{HCl}$ es significativa (valor-p $=0,0058$ ), aunque sólo es ligeramente superior: $0,6 \mathrm{~m}$. De la misma forma, existen diferencias estadísticamente significativas entre el tratamiento con $\mathrm{Al}_{2} \mathrm{O}_{3}$ y el efectuado con $\mathrm{SiC}$ $\mathrm{HF} / \mathrm{HNO}_{3}$ (valor-p $=0,0002$ ), siendo la diferencia mayor que en el caso anterior: 0,9 $\mu \mathrm{m}$. Estos dos resultados confirman que las partículas de $\mathrm{SiC}$ provocan un mayor esfuerzo mecánico sobre la superficie del titanio que las de $\mathrm{Al}_{2} \mathrm{O}_{3}$, por lo tanto, se pueden conseguir valores de rugosidad mayores. Este hecho puede ser debido a una mayor dureza de las partículas de $\mathrm{SiC}$, conclusión que se vería reforzada por su mayor fragilidad, puesta de manifiesto en los ensayos de rotura de partícula. Sin embargo, también cabe señalar que puede ser debida a diferencias en la forma de las partículas, de manera que si las partículas de

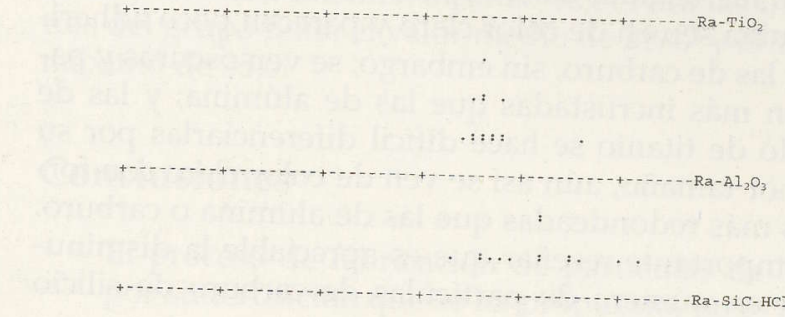

FIG. 6.-Distribución de los valores de RA para los distintos tratamientos de shot peening, variando el tipo de partícula de proyección.

SiC, por ejemplo, son de morfología más aguda que las de $\mathrm{Al}_{2} \mathrm{O}_{3}$, éstas últimas pueden tener un efecto cortante inferior, y en consecuencia, un efecto sobre la superficie del disco, también menor. En cualquier caso, este es un hecho que se debería comprobar con un análisis micrográfico de los distintos tipos de partículas de proyección.

Por su parte, variar el tipo de pasivado no afecta significativamente a la rugosidad en la superficie de los discos (valor-p $=0,2140$ ).

Tabla III. p-valores de las comparaciones de las medias de rugosidad superficial, en discos de Ti C.P., obtenidas con distintos tratamientos de shot peening en función del tipo de partícula de proyección utilizado. Los p-valores se han varianza con tabla ANOVA - si se comparan de t-Student - si se somparan dos tratamientos-y análisis de la estadísticamente significativa si el p-valor es igual o inferior a 0,05 .

\section{Comparación de medias}

$$
\text { p-valor }
$$

Significatividad

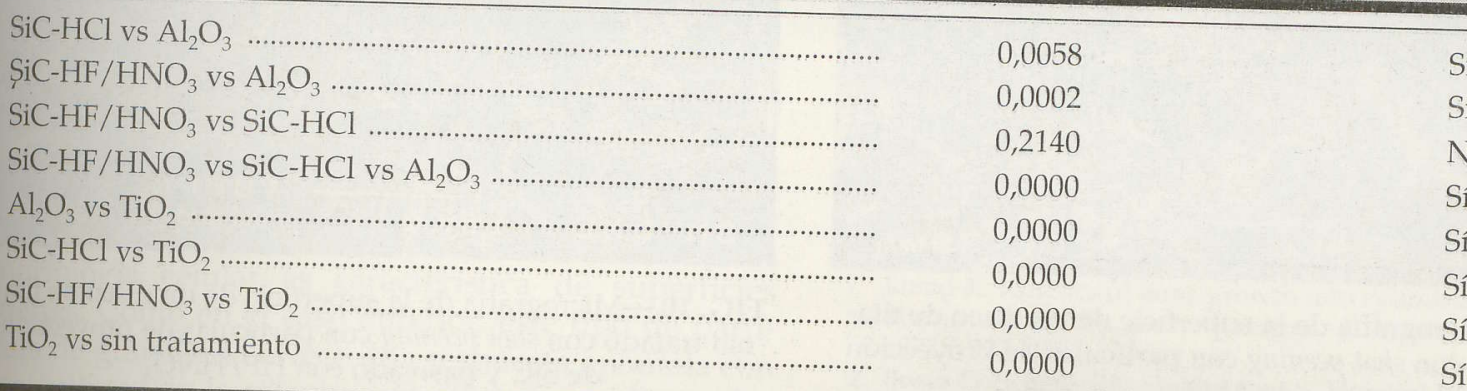


Las imágenes, obtenidas con microscopio electrónico de barrido, de la superficie de los discos tratados con $\mathrm{TiO}_{2}$ (fig. 7); con $\mathrm{Al}_{2} \mathrm{O}_{3}$ (fig. 8); con $\mathrm{SiC}$ y pasivado con $\mathrm{HCl}$ (fig. 9); y con $\mathrm{SiC}$ y pasivado con $\mathrm{HF} / \mathrm{HNO}_{3}$ (fig. 10) refuerzan todos estos resultados. También, en las micrografías, se ven con claridad las partículas procedentes del shot peening que han quedado incrustadas en la superficie de los discos: las de alúmina se ven de color claro y parecen poco adheridas; las de carburo, sin embargo, se ven oscuras y parecen más incrustadas que las de alúmina; y las de óxido de titanio se hace difícil diferenciarlas por su menor tamaño, aún así se ven de color claro con formas más redondeadas que las de alúmina o carburo. Es importante reseñar que es apreciable la disminución en número de partículas de carburo de silicio

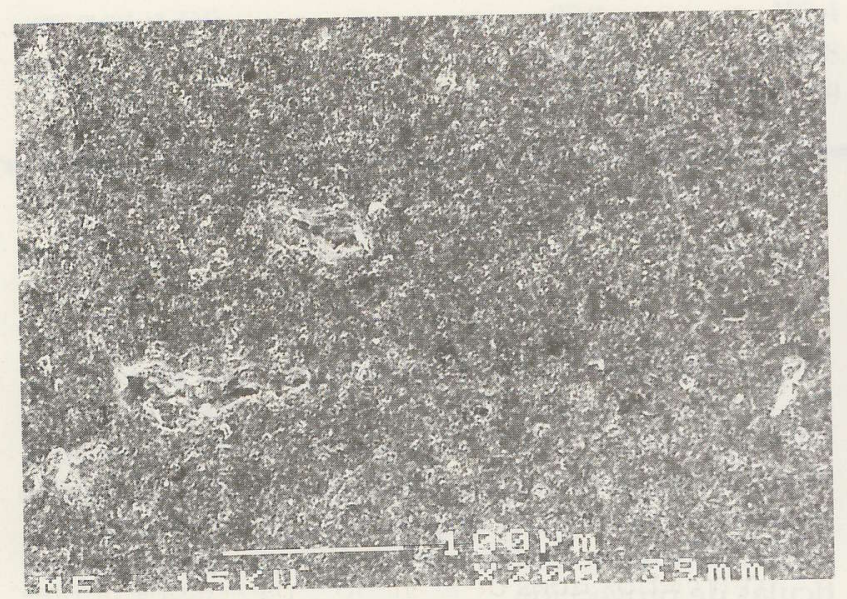

FIG. 7.-Micrografía de la superficie de un disco de titanio tratado con shot peening con partículas de proyección de $\mathrm{TiO}_{2}$.

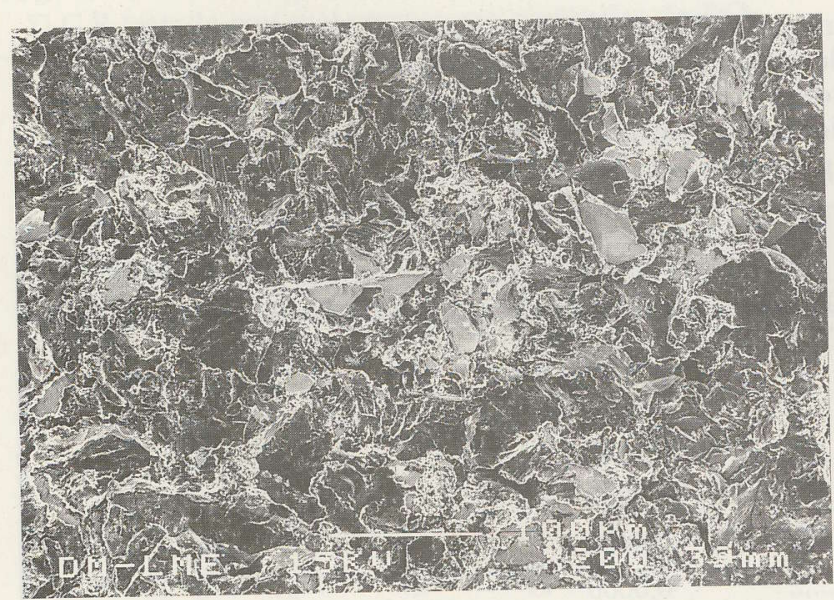

FIG. 8.-Micrografía de la superficie de un disco de titanio tratado con shot peening con partículas de proyección de $\mathrm{Al}_{2} \mathrm{O}_{3}$ que se observan en la micrografía del disco pasivado con $\mathrm{HF} / \mathrm{HNO}_{3}$, respecto a la del pasivado con $\mathrm{HCl}$.

\section{Composición superficial}

Los resultados obtenidos con el microanálisis de energía dispersiva de rayos $\mathrm{X}$ han demostrado que el tratamiento con $\mathrm{TiO}_{2}$ no ha contaminado la superficie del implante con elementos ajenos, como era de esperar. Desde este punto de vista, sigue siendo importante valorar la posibilidad de conseguir el óxido por otras vías para poder realizar el tratamiento del shot peening de forma eficaz mecánicamente.

Por otra parte, no ha habido diferencias entre los porcentajes atómicos de aluminio (tratamiento con

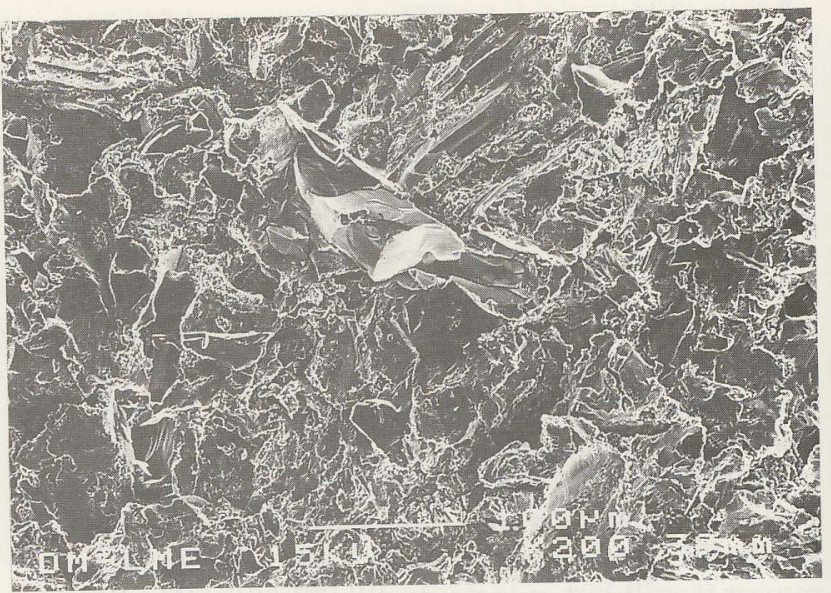

FIG. 9.-Micrografía de la superficie de un disco de titanio tratado con shot peening con partículas de proyección de $\mathrm{SiC}$ y pasivado con $\mathrm{HCl}$.

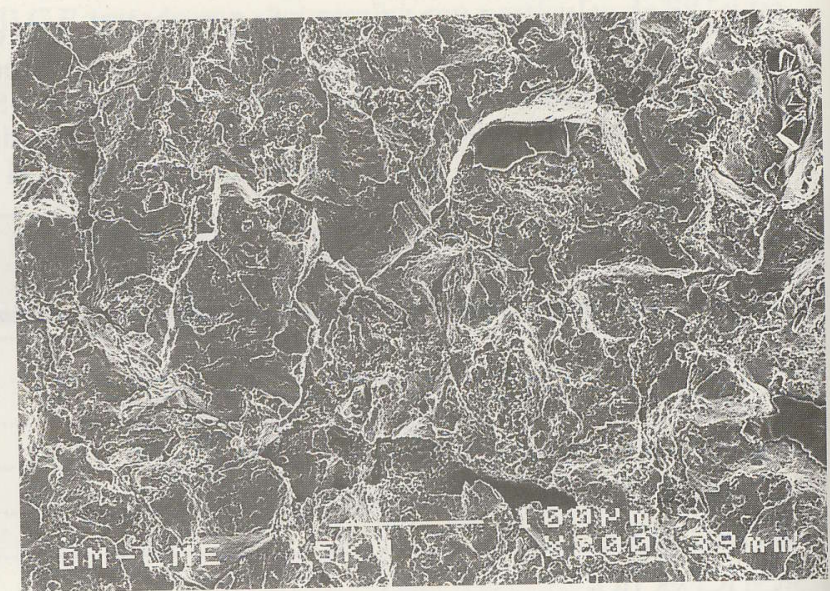

FIG. 10.-Micrografía de la superficie de un disco de titanio tratado con shot peening con partículas de proyección de $\mathrm{SiC}$ y pasivado con $\mathrm{HF} / \mathrm{HNO}_{3}$. 
$\mathrm{Al}_{2} \mathrm{O}_{3}$ ) y silicio (tratamiento con $\mathrm{SiC}$ ) encontrados en la superficie de los discos pasivados con $\mathrm{HCl}$, sin embargo, y este es el resultado más importante de este análisis, el tratamiento de pasivado con $\mathrm{HF} / \mathrm{HNO}_{3}$ en los discos tratados con $\mathrm{SiC}$ reduce, aproximadamente en un $15 \%$, la cantidad de Si en la superficie que deja el pasivado con $\mathrm{HCl}$, en los discos tratados con estas mismas partículas de proyección.

\section{Estudios Celulares}

Ninguno de los materiales presentó signos de citotoxicidad, siendo todos los porcentajes inferiores al $25 \%$, a excepción del grupo de titanio mecanizado, el cual presentó un porcentaje respecto al Control Negativo del 41,2\%. Esta diferencia podría ser debida a que este grupo no había sido pasivado, por lo que aunque fue lavado diversas veces por ultrasonidos, podría ser portador de microimpurezas en su superficie.

El grupo sometido a shot peening con las partículas de $\mathrm{Al}_{2} \mathrm{O}_{3}$ muestra una óptima adherencia celular, estadísticamente significativa respecto al resto de tratamientos $(p<0,05)$. Presenta unos niveles de osteocalcina y proteínas totales superiores a los del grupo del control negativo. Los osteoblastos presentan una morfología de superficies reactivas. El contaje celular fue para el grupo A de un valor medio de 52,8 con un máximo de 59,2 y en el grupo B el valor medio fue 50,07 y el máximo 58,2.

Para las muestras tratadas con CSi y pasivado con $\mathrm{HCl}$ no presenta una adherencia celular tan elevada como el caso de la alúmina; sin embargo ha presentado una concentración de osteocalcina máxima de entre todas las estudiadas y una morfología celular característica de las células diferenciadas sobre superficies reactivas. En cuanto al contaje celular, el valor medio del grupo A fue de 29,67 y el máximo de 31,2 . El grupo B dio un valor medio de 27,40 y un máximo de 36,0 .

El grupo de CSi, pasivado con HF lo situaríamos en el rango de la citocompatibilidad media, pero presenta una alta concentración en osteocalcina así como su morfología celular característica de superficies reactivas. El número presente de células en los cultivos inducidos con vitamina $\mathrm{D}$ ha sido ligeramente superior a los que no presentan vitamina, lo que evidencia una respuesta anómala. Los valores del grupo A fueron de 23,43 como resultado medio y un valor máximo de 29,8 . Los valores medios correspondientes al grupo B fue de 32,20 y un máximo de 39,6.

Las muestras granalladas con $\mathrm{TiO}_{2}$ y pasivadas con $\mathrm{HCl}$ presenta una citocompatibilidad baja, presentando una concentración de osteocalcina media y su morfología celular es característica de superficies inertes. Como en el caso anterior, este tipo de partícula de granallada ofrece una respuesta anómala con los cultivos de la vitamina D. El valor medio en las muestras del grupo A fue 20,93 y el máximo de 24,4. El valor medio de $B$ fue de 28,20 y el máximo de 33,00.

En cuanto al grupo de Ti control sin arenado y sin pasivado muestra una concentración de osteocalcina alta y su morfología celular es característica de superficies inertes. El valor medio del grupo A fue de 27,27 y su valor máximo alcanzó 32,8 . Para las muestras del grupo B fue el valor medio de 29,47 y el valor máximo de 35,0 .

\section{Conclusiones}

- El proceso de fabricación de partículas de $\mathrm{TiO}_{2}$ por sinterización que se ha propuesto, no es adecuado, ya que las partículas obtenidas carecen de las propiedades mecánicas necesarias para su utilización en el shot peening.

- Con la utilización de SiC como partícula de proyección en el proceso de shot peening sobre Ti C.P., se obtiene una rugosidad superficial mayor -aprox. $\Delta \mathrm{Ra}=0,6-0,9 \mu \mathrm{m}$ - que si se utiliza como partícula de proyección el $\mathrm{Al}_{2} \mathrm{O}_{3}$, ambos tipos con tamaños grandes de partícula.

- Después de haber tratado la superficie del Ti C.P. con un proceso de shot peening llevado a cabo con partículas de $\mathrm{SiC}$ de gran tamaño, pasivar la superficie con $\mathrm{HF} / \mathrm{HNO}_{3}$ en lugar de con $\mathrm{HCl}$, conlleva disminuir los porcentajes de $\mathrm{Si}$ en la superficie en aproximadamente un $15 \%$.

- A la vista de los actuales estudios podemos confirmar que los procesos de fijación ósea mejoran con ciertos tipos de arenado y parece que la presencia de ciertos materiales y un adecuado tamaño de rugosidad actúan como estimuladores de la misma. A la espera de estudios más exhaustivos parece ser que los tratamientos con $\mathrm{Al}_{2} \mathrm{O}_{3}$ producen las superficies más adecuadas para el contacto óseo en los implantes dentales de titanio.

\section{Agradecimientos}

Los autores desean agradecer a D. Guillermo Nussbaum y D. Agustín García, de la empresa Materias Primas Abrasivas S.L., la donación del material y equipos con los que se ha llevado a cabo la presente investigación, así como sus comentarios tan valiosos para el desarrollo de este trabajo.

\section{Bibliografía}

1. Predecki P, Stephan JE, Auslaender BA, Mooney VL, Kirkland K. Kinetics of bone growth into cylindrical channels in aluminum oxide and titanium. J Biomed Mater Res. 1972;6:375-400.

2. Buser D, Schenk RK, Steinemann S, Fiorelini JP, Fox CH, Stich $\mathrm{H}$. Influence of surface characteristics on bone integration of ti- 
tanium implants. A histomorphometric study in miniature pigs. J Biomed Mater Res. 1991;25:889-902.

3. Johansson CB, Wenneberg A, Han C-H, Albrektsson T. A quantitative comparison of titanium implants, en Fifth World Biomaterials Congress, Toronto June 1996,p.478.

4. Bowers KT, Keller JC, Michaels CM. Optimitazion of surface micromorphology for enhanced osteoblast response in vitro. Int J Oral Maxillofac Implants. 1992;7:302

5. Groessner-Schreiber B, Tuan RS. Enhanced extracellular matrix production and mineralitazion by osteoblasts cultured on titanium surfaces in vitro. J Cell Sci. 1992;101:209

6. Martin JY, Schwartz Z, Hummert TW, Schraub DM, Simpson I Lankford JrJ, Dean DD, Cochran DL, Boyan BD. Effect of titanium surface roughness on proliferation, differentation, and protein synthesis of human osteoblast-like cells. J Biomed Res. 1995:29:389-401.

7. Oshida Y, Sachdeva R, Miyazaki S, Daly J. Effects of shot peening on surface contact angle of biomaterials 1993. J Mater Sci Mater Med. 4:443-7.
8. Gross U Strunz V. The interface of various glasses and glass ceramics with a bony implantation bed. J Biomed Mater Res 1985:19:251-71.

9. Wenneberg A, Albrektsson T, Andersson B. An animal study of c.p. titanium screws with different surface topographies. J Mater Sci Mater Med. 1995;6:302-9

10. Wenneberg A, Albrektsson T. The influence of surface roughness on implant take, en Fifth World Biomaterials Congress, Toronto June 1996,p.459.

11. Wenneberg A, Albrektsson T, Johansson C, Andersson B. Ex perimental study of turned and grit-blasted screw-shaped implants with special emphasis on effects of blasting material and surface topography. Biomaterials. 1996;17:15-22.

12. NF $990-702$ Matériel médico-chirurgical. Evaluation in vitro de la cytotoxicité des matériaux et dispositifs médicaux

13. Clover J and Gowen M. MG-63 and HOS TE 85. Human Osteosarcoma Cell Lines Representative Models of the Osteoblastic Phenotype? Bone. 1994,15(6):585-91

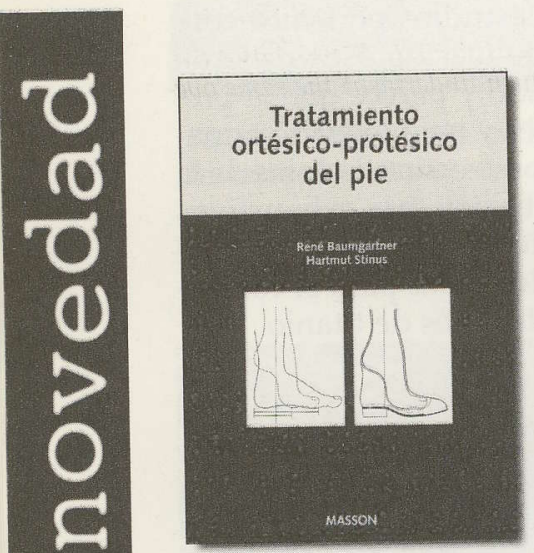

Un tomo $(17 \times 24 \mathrm{~cm})$ con 236 páginas aprox y 297 ilustraciones. y 297 in Encuadernado en tapa dur. ISBN: 84-458-0432-4 ○1997

\section{INDICE DE CAPITULOS}

\section{PARTE I FUNDAMENTOS}

I. Anatomía funcional y biomecanica del pié

2. Evólución del pie del niño

3. Desarrollo del pie

4. Estática y dinámica del pie. Marcha normal 28

5. Técnicas de exploración

6. Mediciones electrónicas de la distribución

$$
\text { de presiones }
$$

PARTE II. PATOLOGIA DEL PIE

Y SU TRATAMIENTO ORTOPÉDICO

7. Pie planovalgo

8. Pie plano transverso

9. Pie cavo

10. Pie equino

11. Pie equinovaro

12. Pie aducto

13. Pie talo

\section{Tratamiento ortésico-protésico del pie \\ R. Baumgartner y \\ H. Stinus}

Obra de carácter práctico que, estructurado en 4 partes, profundiza en aquellos aspectos de consulta diaria en ortopedia.

Parte I. Aspectos fundamentales básicos: anatomía, biomecánica, crecimiento y dinámica, técnica de exploración.

Parte II. Las alteraciones patológicas y alternativas terapéuticas posibles.

Parte III. La técnica ortopédica del calzado.

Parte IV. El tratamiento conservador correspondiente.

14. Alteraciones durante el crecimiento, anomalias del esqueleto y exostosis

15. Pie postraumático

16. Pie reumático

17. Pie en là parálisis cerebral

18. Pie diabético (neuropático)

19. Dismetrías en las extremidades inferiores 105 . Lesiones deportivas y de estrés en el pie y

$$
\text { la pierna }
$$

21. Hallux valgus, dedos en martillo y en garra 125

22. Dedos en martillo: ortesis correctoras de

23. Amputación y tratamiento con prótesis ..135

PARTE III. CALZADO

Y TÉCNICA ORTOPÉDICA

24. El pie y su calzado desde el punto de vistá mitólogico e historicocultura

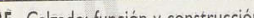

26. Calzado infantil

27. Conceptos sobre técnica del calzado ortopédico

28. Plantillas ortopédicas

29. Aiustes ortopédicos en el calzado de serie 179

30. Calzado de vendaje y calzado terapéutico 186

31. Calzado ortopédico a medida .........19

32. Calzado interno ortopédico ........ 198

33. Ortesis para el retropié _..........201

PARTE IV TRATAMIENTO

CONSERVADOR

34. Diagnóstico y tratamiento manuales del pie (quirodiagnóstico y quiroterapia)

35. Fisioterapia del pie

36. Profillaxis del pie

Índice alfabético de materias
THRJETH DE PE D

$\checkmark$ Sí, deseo remitan a mi nombre esta o

BAUMGARTNER: TRATAMIENTO ORTÉSICO-PROTÉSICO DEL PIE

PVP: 8.850 Ptas. con IVA y 8.510 sin

FORMA DE PAGO

Contra reembolso sin cargo alquno.

Nombre

$1^{\text {er Apellido }}$

$2^{\circ}$ Apellido

Año nacimiento

Teléfono

Especialidad

Dirección

Localidad

FIRMA
MASSON, S.A.

Ronda General Mitre, 149

08022 BARCELONA (España) Fax: 93-253 0515

e-mail: grupo.masson@ben.servicom.es 